\title{
A branch-and-cut algorithm for a multi-item inventory distribution problem *
}

\author{
Agostinho Agra ${ }^{1}$, Adelaide Cerveira ${ }^{2}$, and Cristina Requejo ${ }^{1}$ \\ 1 University of Aveiro and CIDMA, 3810-193 Aveiro, Portugal, \\ \{aagra, crequejo\}@ua.pt \\ 2 University of Trás-os-Montes e Alto-Douro, 5001-801 Vila Real, and INESC TEC, Portugal \\ cerveira@utad.pt
}

\begin{abstract}
This paper considers a multi-item inventory distribution problem motivated by a practical case occurring in the logistic operations of an hospital. There, a single warehouse supplies several nursing wards. The goal is to define a weekly distribution plan of medical products that minimizes the visits to wards, while respecting inventory capacities and safety stock levels. A mathematical formulation is introduced and several improvements such as tightening constraints, valid inequalities and an extended reformulation are discussed. In order to deal with real size instances, an hybrid heuristic based on mathematical models is introduced and the improvements are discussed. A branch-and-cut algorithm using all the discussed improvements is proposed. Finally, a computational experimentation is reported to show the relevance of the model improvements and the quality of the heuristic scheme.
\end{abstract}

Keywords: Multi-item inventory; Hospital logistics; Branch-and-cut; Supply chain management.

\section{Introduction}

We consider a Multi-Item Inventory Distribution (MIID) problem. The motivation for this study is to investigate logistics activities in respect to supply chain management in an hospital. There is a central warehouse that receives goods from suppliers and delivers those goods to nursing wards regularly. The ward's demand comprise a wide variety of products which include, amongst others, medicines, hospital supplies and medical devices. The main focus of this work is the planning of the delivery scheme of goods to meet the demand while keeping the stock levels between the desired bounds. The planning horizon is one week and should be repeated every week. The MIID problem is to find the delivery plan that satisfies the inventory and delivery capacity constraints that minimizes the number of visits to wards.

From the inventory management point of view, we consider a multi-item problem where one warehouse supplies several locations (wards), which are typically retailers in literature. Each location is visited at most once per day (except for extraordinary deliveries not considered here). As the number of time periods is small, inventory aspects are considered only at the supplied locations, since the supply decisions for the warehouse are taken with months in advance. Only a part of items (goods) are consumed at each location. For those items, safety stocks are mandatory at each location. Furthermore, the stock level in the first and last period

\footnotetext{
* Published in Machine Learning, Optimization, and Big Data: Second International Workshop, MOD 2016, Revised Selected Papers. Lecture Notes in Computer Science, Volume 10122, 144-158, 2016.
} 
must coincide, in order to ensure that the delivery plan can be replicated in subsequent weeks. A global stock upper bound is imposed at each location. Given the proximity of locations, the routing aspects can be ignored. Only the capacity of the vehicle is considered to bound the amount to be delivered in each time period.

Operations Research has been widely used in health care issues. Many operations research papers on health care subjects have been published in both Operations Research and Health Care journals. In [19] is presented a survey which points out a variety of studied problems in health care using operational research techniques. They include, among others, health care planning aspects, management and logistics health problems and the health care practices with treatment planning and preventive care. In [23] is presented the state-of-the-art of the research on material logistics management in an hospital. In [14,22] are considered pharmaceutical supply chain and inventory management issues in health care industry. The same topic of research is covered in [9] where the tasks and management approaches of hospital materials are considered. In [16] a case study is reported that looks into logistics activities of hospitals in Singapore. From the point of view of inventory management, complex problems combining inventory management and distribution decisions are receiving an increased attention in the last years. In [10] an overall introduction into inventory management is presented. In [7] a review of replenishment policies for hospital inventory systems and two models to deal with capacity limitations and service requirements are presented. In [18] inventory models are discussed for hospital problems. Practical complex inventory problems occur in other industries, from maritime transportation [2,3] to land transportation [8]. A recent review on complex inventory problems combined with production and distribution aspects is given in [1]. In relation to other practical problems, the problem considered in this paper has the particularity of including a huge number of variables and the distribution costs are negligible.

We provide a mixed integer formulation for MIID problem and discuss several approaches to improve that formulation, such as, tightening of constraints; derivation of valid inequalities; use of an extended reformulation. Several such techniques have been used for related inventory management problems and are of main importance to allow the improved formulation to solve practical problems through branch-and-cut and branch-and-bound algorithms. For exact branch-and-cut approaches see [2,3,5,20]. The MIID problem is NP-hard, since it generalizes well-known NP-hard problems, such as the capacitated lot-sizing problem [17]. Therefore we also propose an heuristic scheme to find good feasible solutions. This scheme works in two steps. In the first step we solve a relaxation obtained by aggregating all products and ignoring some constraints. In the second step a local search is conducted in the neighborhood of the first step solution. Finally, an exact branch-and-cut algorithm is proposed to the MIID problem. This algorithm uses the improved formulation and it is feeded up with the upper bound given by the heuristic scheme.

The outline of the paper is as follows. In Section 2 we introduce a mixed integer formulation to the MIID problem. In Section 3 we discuss the model improvements. In Section 4 we introduce the heuristic scheme and in Section 5 a branch-and-cut algorithm based on the improved model is proposed. In Section 6 the details of benchmark instances generation are given, and computational results to test the model, the model improvements, the heuristic scheme and the branch-and-cut algorithm are presented. Finally, the conclusions are stated in Section 7. 


\section{Mathematical model}

In this section we introduce a mixed integer formulation to the MIID problem. Consider the sets $N=\{1, \ldots, n\}, L=\{1, \ldots, m\}$, and $T=\{1, \ldots, r\}$, representing the set of items, the set of locations (wards) to visit, and the set of time periods, respectively. Additionally, $N_{j}$ represents the set of items consumed in location $j$ and $L_{i}$ represents the set of locations that consume item $i$. We define the following variables: $x_{i j t}$ is the amount of item $i$ to be delivered at location $j$ in time period $t ; s_{i j t}$ is the stock level of item $i$ in location $j$ at time period $t$; and the binary variables $y_{j t}$ take value 1 if there is a delivery in location $j$ at time period $t$, and 0 otherwise. Additionally, we consider the following parameters. The demand for item $i$ in location $j$ at time period $t$ is represented by $d_{i j t}$. The minimum stock level (safety stock) of item $i$ in location $j$ at time period $t$ is $\underline{S}_{i j t}$. There is a single vehicle with capacity $C$, and we assume item $i$ uses $c_{i}$ units of that capacity. We also assume that only $K$ locations can be visited per time period, and each location has a limited stock capacity of $V_{j}$ items. The initial stock level of product $i$ at location $j$ is given by $S_{i j} . M$ is an upper-bound for the number of items delivered at each location and each period. The MIID model is as follows:

$$
\begin{aligned}
& \min \quad \sum_{j \in L} \sum_{t \in T} y_{j t} \\
& \text { s.t. } \quad x_{i j t}+s_{i j, t-1}=d_{i j t}+s_{i j t}, \quad i \in N, j \in L, t \in T, \\
& \sum_{i \in N_{j}} x_{i j t} \leq M y_{j t}, \quad j \in L, t \in T, \\
& \sum_{j \in M} y_{j t} \leq K, \quad t \in T, \\
& \sum_{i \in N} \sum_{j \in L_{i}} c_{i} x_{i j t} \leq C, \quad t \in T, \\
& \sum_{i \in N_{j}} s_{i j t} \leq V_{j}, \quad j \in L, t \in T, \\
& s_{i j t} \geq \underline{S}_{i j t}, \quad i \in N, j \in L_{i}, t \in T, \\
& s_{i j 0}=S_{i j}, \quad i \in N, j \in L_{i} \text {, } \\
& x_{i j t} \in \mathbb{Z}_{+} \text {, } \\
& i \in N, j \in L_{i}, t \in T \text {, } \\
& y_{j t} \in\{0,1\} \text {, }
\end{aligned}
$$

The objective function (1) is to minimize the total number of visits to locations. Constraints (2) are the inventory flow balance at the locations. Constraints (3) ensure that if any item is delivered to location $j$ at period $t$, then variable $y_{j t}$ must be one. Constraints (4) and (5) impose limits on the deliveries in each time period. Constraints (4) state that only a limited number of locations can be visited while the vehicle capacity is guaranteed by constraints (5). Constraints (6) impose a storage capacity limit at each location. Safety stocks are imposed by the inventory lower bound constraints (7). Constraints (8), (9) and (10) are the variable domain constraints.

In this practical case it is desirable that the distribution plan for the planning week could be repeated for other weeks with similar demand rates. For this purpose, we need to ensure that the stock at the end of the time horizon is equal to the initial stock, that is, $\underline{S}_{i j r}=S_{i j}$. 
Example 1. In Table 1 we present an example having 5 items, 5 periods and 2 locations (wards) with $N_{1}=\{1,2,3,4\}$ and $N_{2}=\{2,4,5\}$. The optimal delivery quantities are presented in the last five columns with $y_{12}=y_{13}=y_{24}=y_{25}=1$ and the optimal value is 4 .

Table 1. Example of a supply scheme. For each item and each ward is displayed the initial stock, and for each time period is displayed the safety stock, the demand and the optimal delivery quantities.

\begin{tabular}{|c|c|c|c|c|c|c|c|c|c|c|c|c|c|c|c|c|c|}
\hline \multirow{2}{*}{$\begin{array}{c}\text { warc } \\
(j)\end{array}$} & \multirow{2}{*}{$\begin{array}{c}\text { item } \\
(i)\end{array}$} & \multirow{2}{*}{$S_{i j}$} & \multicolumn{5}{|c|}{$\underline{S}_{i j t}$} & \multicolumn{5}{|c|}{ demand $\left(d_{i j t}\right)$} & \multicolumn{5}{|c|}{ deliver $\left(x_{i j t}\right)$} \\
\hline & & & $t=1$ & $t=2$ & $t=3$ & $t=4$ & $t=5$ & $t=1$ & $t=2$ & $t=3$ & $t=4$ & $t=5$ & $t=1$ & $t=2$ & $t=3$ & $t=4$ & $t=5$ \\
\hline \multirow{4}{*}{1} & 1 & 7 & 2 & 2 & 2 & 2 & 7 & 3 & 3 & 5 & 2 & 9 & - & 22 & - & - & - \\
\hline & 2 & 44 & 11 & 11 & 11 & 11 & 44 & 7 & 8 & 9 & 10 & 21 & - & 19 & 36 & - & - \\
\hline & 3 & 27 & 7 & 7 & 7 & 7 & 27 & 4 & 7 & 6 & 4 & 12 & - & 33 & - & - & - \\
\hline & 4 & 25 & 6 & 6 & 6 & 6 & 25 & 4 & 2 & 4 & 3 & 18 & - & 31 & - & - & - \\
\hline \multirow{3}{*}{2} & 2 & 37 & 9 & 9 & 9 & 9 & 37 & 5 & 6 & 8 & 10 & 18 & - & - & - & 2 & 45 \\
\hline & 4 & 27 & 7 & 7 & 7 & 7 & 27 & 5 & 3 & 5 & 2 & 18 & - & - & - & - & 33 \\
\hline & 5 & 23 & 6 & 6 & 6 & 6 & 23 & 3 & 2 & 4 & 2 & 18 & - & - & - & 28 & 1 \\
\hline
\end{tabular}

\section{Model improvements}

It is well-known that the performance of exact algorithms based on mathematical models, such as the branch-and-bound and the branch-and-cut depend greatly on the quality of the model, see [15] for general integer programming problems, and [17] for lot-sizing problems. In this section we discuss model improvements that aim at tightening the formulation, that is, deriving a formulation whose linear relaxation is closer to the convex hull of the set of feasible solutions.

In order to ease the presentation we start with the introduction of the concept of net demand (see [2] for a related use of this concept). The net demand of item $i$ in location $j$ at time period $t$ is denoted by $n d_{i j t}$ and represents the minimum amount of item $i$ that must be delivered in location $j$ at time period $t$ taking into account the initial stock level and the safety stocks (lower bound on the inventory levels). In this case it can be computed as follows:

$$
n d_{i j t}=\max \left\{0, \sum_{\ell=1}^{t} d_{i j \ell}+\underline{S}_{i j t}-S_{i j}-\sum_{\ell=1}^{t-1} n d_{i j \ell}\right\} .
$$

The net demand is computed iteratively for each time period. For time period $t=1$, the net demand is just the demand in period $1, d_{i j 1}$, plus the safety stock at the end of time period $1, \underline{S}_{i j 1}$, minus the initial inventory level. If the initial inventory level is enough to cover the demand and the safety stock, then the net demand is zero. For the remaining periods, the net demand is computed as the net demand until period $t\left(\sum_{\ell=1}^{t} d_{i j \ell}+\underline{S}_{i j t}-S_{i j}\right)$, minus the accumulated net demand until the previous time period $\left(\sum_{\ell=1}^{t-1} n d_{i j \ell}\right)$.

Example 2. Consider the case of item 1 and location 1 given in Example 1, where the initial inventory level is $S_{11}=7$, the safety stock vector for 5 periods is given by $\underline{S}_{11}=[2,2,2,2,7]$, and the demand vector is $d_{11}=[3,3,5,2,9]$. The net demands are given by: $n d_{111}=$ $\max \{0,3+2-7\}=0, n d_{112}=\max \{0,6+2-7-0\}=1, n d_{113}=\max \{0,11+2-7-1\}=5$, $n d_{114}=\max \{0,13+2-7-6\}=2, n d_{115}=\max \{0,22+7-7-8\}=14$. 
Net demands can be used to strengthen inequalities (3). Constant $M$ can be replaced by

$$
M_{j t}=\sum_{i \in N_{j}} \sum_{\ell=t}^{r} n d_{i j \ell}
$$

which is the minimum upper bound on the delivery quantity at each location $j$ and on the time period $t$. The strengthened inequalities are

$$
\sum_{i \in N_{j}} x_{i j t} \leq M_{j t} y_{j t}, \quad j \in L, t \in T .
$$

Next, we discuss two types of approaches to further strengthen the model: the inclusion of valid inequalities and the use of extended formulations.

\subsection{Valid inequalities}

A first family of valid inequalities can be derived by disaggregation of inequalities (11) (or the weaker version (3)), as follows:

$$
x_{i j t} \leq \sum_{\ell=t}^{r} n d_{i j \ell} y_{j t}, \quad i \in N, j \in L_{i}, t \in T .
$$

As a large number of items may be considered, introducing all such inequalities can make the model too large. Several approaches are possible. An approach is to add these inequalities dynamically, that is, add each inequality when it is violated by the linear fractional solution. Another approach is to consider only a representative item for each location and add these inequalities for this item. For each location $j \in L$, we choose the representative item, denoted by $i(j)$, as follows:

$$
i(j)=\operatorname{argmax}_{i \in N_{j}}\left\{\sum_{t \in T} n d_{i j t}\right\} .
$$

Another family of valid inequalities is a type of cut set inequalities which have been introduced for related, although simpler, lot-sizing problems with upper bounds on the inventory levels, see [6]. For each $j \in L$ define the set $T_{j}=\left\{\left(t_{1}, t_{2}\right) \in(T, T) \mid \sum_{i \in N_{j}} \sum_{t=t_{1}}^{t_{2}} n d_{i j t}>V_{j}\right\}$. Thus $\left(t_{1}, t_{2}\right)$ belongs to $T_{j}$ if the accumulated net demand during the periods $t_{1}$ to $t_{2}$ is greater than the upper stock capacity, which implies that this demand cannot be fully met using only inventory. Thus, at least a delivery is necessary to the location $j$ during the periods $t_{1}$ to $t_{2}$ :

$$
\sum_{t=t_{1}}^{t_{2}} y_{j t} \geq 1, \quad j \in L,\left(t_{1}, t_{2}\right) \in T_{j} .
$$

When $t_{1}=1$ we obtain a particular case since the initial inventory level can be used instead of the upper bound capacity $V_{j}$. As we are already using this initial stock to compute the net demands, we just need to check in which time period at least one net demand is positive in order to enforce the first visit to that location. For each location $j$ let $t(j)$ denote the first time period where the net demand is positive, that is, $t(j)=\min \left\{t \in T \mid \sum_{i \in N_{j}} \sum_{\ell=1}^{t-1} n d_{i j \ell}=\right.$ $\left.0 \wedge \sum_{i \in N_{j}} \sum_{\ell=1}^{t} n d_{i j \ell}>0\right\}$. Then, the following inequalities are valid for the set of feasible solutions of the MIID problem:

$$
\sum_{\ell=1}^{t(j)} y_{j \ell} \geq 1, \quad j \in L
$$




\subsection{Extended formulation}

A common approach to derive tighter models in lot-sizing problems is to use extended formulations that use additional variables, see for instance [11,20,21]. Here we consider additional variables $v_{i j t \ell}$ to indicate the fraction of the net demand for item $i$ in location $j$ at time period $\ell$ that is delivered in time period $t$, for $\ell \geq t$.

The following constraints are added to the model MIID:

$$
\begin{array}{ll}
x_{i j t}=\sum_{\ell=t}^{r} n d_{i j \ell} v_{i j t \ell}, & i \in N, j \in L_{i}, t \in T, \\
v_{i j t \ell} \leq y_{j t}, & i \in N, j \in L_{i}, t, \ell \in T \mid t \leq \ell, \\
\sum_{t=1}^{\ell} v_{i j t \ell}=1, & i \in N, j \in L_{i}, \ell \in T, \\
v_{i j t \ell} \geq 0, & i \in N, j \in L_{i}, t, \ell \in T \mid t \leq \ell .
\end{array}
$$

Constraints (16) relate the new variables with the $x_{i j t}$ variables. Constraints (17) ensure that if $v_{i j t \ell}>0$ for at least one $\ell \geq t$, then $y_{j t}$ must be one. Constraints (18) ensure that demand must be completely satisfied, and constraints (19) define the new variables as nonnegative. Constraints (18) together with constraints (19) ensure $v_{i j t \ell} \leq 1$.

The resulting model (with constraints (2)-(10), (16)-(19)), denoted by MIID-EF, can become too large for practical size instances. In order to use such type of reformulation in practical instances we tested two weaker approaches. One is to introduce constraints (16)(19) only for the representative item $i(j)$ of each location $j$. The resulting model will be denoted by MIID-EF-R. The other approach is to introduce the extended formulation for the aggregated model. For the aggregated case we define $V_{j t \ell}$ to indicate the fraction of the total net demand for period $\ell$ in location $j$ delivered in time period $t$, for $\ell \geq t$. The extended formulation for the aggregated model, denoted by MIID-A-EF, is given by (2)-(10), (20)-(23), with:

$$
\begin{array}{ll}
\sum_{i \in N_{j}} x_{i j t}=\sum_{i \in N_{j}} \sum_{\ell=t}^{r} n d_{i j \ell} V_{j t \ell}, & j \in L, t \in T, \\
V_{j t \ell} \leq y_{j t}, & j \in L, t, \ell \in T \mid t \leq \ell, \\
\sum_{t=1}^{\ell} V_{j t \ell}=1, & j \in L, \ell \in T, \\
V_{j t \ell} \geq 0, & j \in L, t, l \in T \mid t \leq \ell .
\end{array}
$$

\section{Heuristic Scheme}

As the MIID problem is NP-hard it is important to derive heuristic schemes that provide good quality solutions within reasonable amount of time. Here we describe an hybrid heuristic scheme that uses mathematical models. Hybrid schemes have been successfully employed in the past [4].

First we solve a relaxation of MIID-A-EF where the integrality of the $x$ variables is relaxed (constraints (9)) and the vehicle capacity constraints (5) are ignored. This relaxation is tightened with inequalities (15). This relaxation of the MIID problem is denoted RMIID. 
A feasible solution $(\bar{x}, \bar{y})$ to the RMIID problem may lead to an infeasible solution to the MIID problem. That is, fixing $y_{j t}=\bar{y}_{j t}$ in model (2)-(10) may originate an infeasible solution. Nevertheless such solution is expected to be "close" to a good solution of MIID. The next step is a local search heuristic that searches for solutions in a neighborhood of $\bar{y}$. In order to do that search we add the following inequality,

$$
\sum_{j \in L, t \in T \mid \bar{y}_{j t}=0} y_{j t}+\sum_{j \in L, t \in T \mid \bar{y}_{j t}=1}\left(1-y_{j t}\right) \leq \Delta .
$$

Inequality (24) counts the number of variables that are allowed to flip their value from the value taken in the solution.

We denote the Local Search model which is the MIID model with the additional constraint (24) by $\operatorname{LSP}(\Delta)$. Notice that by increasing the value of $\Delta$ the model becomes less restrictive, that is, the neighborhood becomes larger. For larger values of $\Delta$ the constraint (24) becomes ineffective and we obtain the original model MIID. This local search can be seen as a particular case of the Local Branching approach introduced in [13].

The heuristic scheme is given in Algorithm 1.

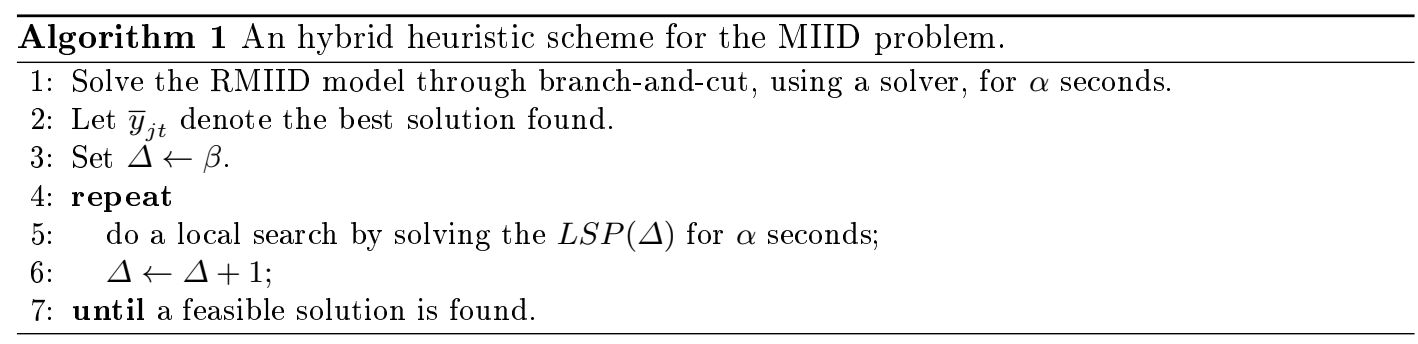

In the computational tests we used $\alpha=100$ and $\beta=5$.

\section{Branch and cut algorithm}

The discussed model improvements to tighten the formulation and the proposed heuristic were used together within a branch and cut algorithm. Several combinations were tested. For completeness, the exact approach that provided best results is given below in Algorithm 2.

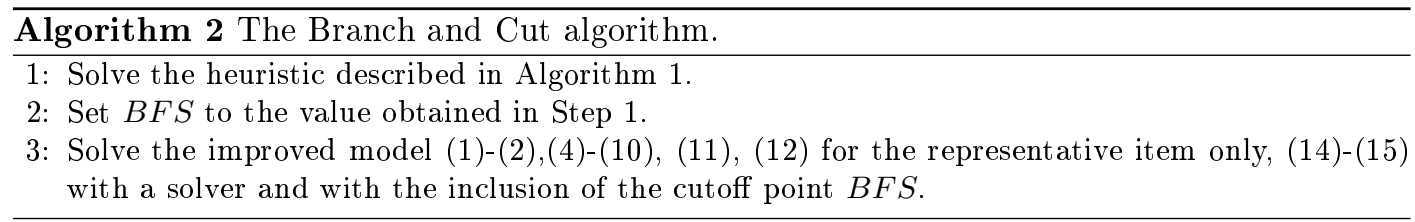

\section{Computational Results}

In order to test the performance of the heuristic and the exact approaches, in this section we report some computational experiments. All the computational tests were performed using a processor Intel(R) Core(TM) i7-4750HQ CPU @ $2.00 \mathrm{GHz}$ with $8 \mathrm{~GB}$ of RAM and using the software Xpress 7.6 (Xpress Release July 2015 with Xpress-Optimizer 28.01.04 and XpressMosel 3.10.0) [12]. 
First we provide details on the generation of benchmark instances. We generated 12 instances with $n=4200$ items and $m=20$ wards that resemble the dimensions of the real problem. The demands were randomly generated following patterns of some representative items. Except in special cases, there are no deliveries on weekends and holidays. Thus we considered $r=5$ time periods. The first four periods are a single week day, corresponding to Monday, Tuesday, Wednesday and Thursday. The last one, Friday, is deemed to correspond to three days, Friday, Saturday and Sunday. There are items required in all nursing wards and there are specific items that are only required in some nursing wards. The data was generated such that approximately $0.6 \%$ of the items are required in all the nursing wards and each ward requires about $10 \%$ of the items. The demand of each item, at each ward on the first four periods are randomly generated between 0 and 60 and in the fifth period can be up to 180. The capacity $c_{i}$ of each item $i$ is a random number between 1 and 5 and the capacity $C$ of the vehicle is approximately $30 \%$ of the capacity of the total demand during all the five periods. The safety stock $\underline{S}_{i j t}$ is obtained by rounding up the average demand by period. For the initial stock level $S_{i j}$ of each product $i$ at location $j$ two cases were considered. In case 1 this value is approximately four times the corresponding average demand per period while in case 2 this value is approximately three times of the average demand. The value of stock capacity $V_{j}$ is a randomly generated number between $30 \%$ and $80 \%$ of the total demand at this nursing ward plus the initial stock level. These instances can be found in http://www.cerveira.utad.pt/datamod2016.

In the computational experiments were considered two values for parameter $K, K=15$ and $K=12$, which bounds the number of locations that can be visited per time period, constraints (4). The instances $A 11, A 21, A 31, A 12, A 22, A 32$ consider parameter $K=15$ while instances $B 11, B 21, B 31, B 12, B 22, B 32$ consider parameter $K=12$. Instances ending with 1 correspond to case 1 of the initial stock level while instances ending with 2 correspond to case 2 of the initial stock level. For each pair of these parameters we have three instances. The middle number of the name of the instance differentiates these three instances.

Table 2. Optimal supply scheme

\begin{tabular}{|c|c|c|c|c|c|c|c|c|c|c|c|c|}
\hline & \multicolumn{6}{|c|}{ case 1} & \multicolumn{6}{|c|}{ case 2} \\
\hline ward & $t=1$ & $t=2$ & $t=3$ & $t=4$ & $t=5$ & \# visits & $t=1$ & $t=2$ & $t=3$ & $t=4$ & $t=5$ & \# visits \\
\hline w1 & & & $\checkmark$ & & $\checkmark$ & 2 & $\checkmark$ & & & & $\checkmark$ & 2 \\
\hline w2 & $\checkmark$ & & & & $\checkmark$ & 2 & $\checkmark$ & & & $\checkmark$ & & 2 \\
\hline w3 & & $\checkmark$ & & & & 1 & $\checkmark$ & $\checkmark$ & & & & 2 \\
\hline $\mathrm{w} 4$ & & $\checkmark$ & & & & 1 & & $\checkmark$ & & & & 1 \\
\hline w5 & $\checkmark$ & & & & $\checkmark$ & 2 & $\checkmark$ & & & & $\checkmark$ & 2 \\
\hline w6 & & & $\checkmark$ & & & 1 & & $\checkmark$ & & & $\checkmark$ & 2 \\
\hline w7 & & $\checkmark$ & & & & 1 & & $\checkmark$ & & & & 1 \\
\hline w8 & $\checkmark$ & & & & $\checkmark$ & 2 & $\checkmark$ & & & & $\checkmark$ & 2 \\
\hline w9 & $\checkmark$ & & & & $\checkmark$ & 2 & & $\checkmark$ & & & $\checkmark$ & 2 \\
\hline \begin{tabular}{|l|} 
w10 \\
\end{tabular} & & & $\checkmark$ & & & 1 & $\checkmark$ & & & & $\checkmark$ & 2 \\
\hline \begin{tabular}{|l|} 
w11 \\
\end{tabular} & $\checkmark$ & & & $\checkmark$ & & 2 & $\checkmark$ & & & $\checkmark$ & & 2 \\
\hline w12 & & $\checkmark$ & & & $\checkmark$ & 2 & $\checkmark$ & & & & $\checkmark$ & 2 \\
\hline w13 & & $\checkmark$ & & $\checkmark$ & & 2 & & $\checkmark$ & & $\checkmark$ & & 2 \\
\hline w14 & & $\checkmark$ & & & $\checkmark$ & 2 & & $\checkmark$ & & & $\checkmark$ & 2 \\
\hline \begin{tabular}{|l|}
$\mathrm{w} 15$ \\
\end{tabular} & $\checkmark$ & & & $\checkmark$ & & 2 & $\checkmark$ & & & & $\checkmark$ & 2 \\
\hline \begin{tabular}{|l|} 
w16 \\
\end{tabular} & & & $\checkmark$ & & & 1 & $\checkmark$ & $\checkmark$ & & & & 2 \\
\hline w17 & $\checkmark$ & & & & $\checkmark$ & 2 & & $\checkmark$ & & & $\checkmark$ & 2 \\
\hline w18 & & $\checkmark$ & $\checkmark$ & & & 2 & & $\checkmark$ & & & $\checkmark$ & 2 \\
\hline w19 & $\checkmark$ & & & $\checkmark$ & & 2 & & $\checkmark$ & & $\checkmark$ & & 2 \\
\hline \multirow[t]{3}{*}{ w20 } & $\checkmark$ & & & & $\checkmark$ & 2 & & $\checkmark$ & & & $\checkmark$ & 2 \\
\hline & \multicolumn{6}{|c|}{ Total delivers $=34$} & \multirow{2}{*}{\multicolumn{6}{|c|}{ Total delivers $=38$}} \\
\hline & & Aver & age of & $S_{i j}=$ & $=124$. & & & & & & & \\
\hline
\end{tabular}


In Table 2 are shown the days when there are deliveries for an optimal solution considering the two cases for initial stock level. This illustrates how the initial stock levels influence the weekly number of deliveries. In this instance, the number of items required at each nursing ward ranges between 409 and 477, being 436 on average. Amongst the 4200 items, 28 are required in all wards and approximately $45 \%$ are specific items of a nursing ward. In case 1 , the initial stock $S_{i j}$ on average is equal to 124.46 and the optimal value is 34 while, in case 2, the average initial stock is equal to 93.36 and the optimal value decreased to 38 . With higher values of initial stock, case 1, there are more wards visited only once. By a detailed analysis of the solutions it can be seen that even in the cases with two delivers per week, there are some products delivered once.

The computational results obtained for the 12 instances of the MIID problem are displayed in Tables 3, 5, 6, and 7. All the computational times are given in seconds.

Table 3 displays the computational results for the model with no improvements (model (1)(10)) solved using the branch-and-cut procedure from the Xpress solver, with a time limit of 7200 seconds. Column " $b b$ " indicates the value of the best bound found when the algorithm terminates. The corresponding gap, gap $=\frac{m i p-b b}{m i p} \times 100$, is displayed in column "gap", and the value of the best integer solution found is indicated in column " $m i p$ ". We can see that for all the instances the algorithm has stopped without proving optimality.

Table 3. Computational results for the model (1)-(10) solved using the branch-and-cut procedure.

\begin{tabular}{|c|c|c|c|c|c|c|c|}
\hline instance & $b b$ & gap & mip & instance & $b b$ & gap & mip \\
\hline A11 & 29.0 & 12.0 & 33.0 & B11 & 31.2 & 8.2 & 34.0 \\
\hline A 21 & 30.2 & 18.3 & 37.0 & B21 & 31.2 & 15.7 & 37.0 \\
\hline A31 & 29.1 & 17.0 & 35.0 & B31 & 30.0 & 16.6 & 36.0 \\
\hline A12 & 26.5 & 30.1 & 38.0 & B12 & 28.3 & 25.6 & 38.0 \\
\hline $\mathrm{A} 22$ & 30.4 & 21.9 & 39.0 & B22 & 32.2 & 17.3 & 39.0 \\
\hline A32 & 27.6 & 25.4 & 37.0 & B32 & 38.0 & 4.9 & 40.0 \\
\hline
\end{tabular}

Tables 5 and 6 display the computational results for the linear relaxation of model (1)(10), denoted by lp, and its improvements from Section 3. For readability, in Table 4 the tested relaxations are summarized and characterized in terms of their constraints.

Table 4. List of relaxed models with indication of the constraints added to the linear relaxation lp.

\begin{tabular}{cl}
\hline relaxed model & added constraints \\
\hline lp-A & $(11)$ \\
lp-D & $(12)$ \\
lp-R & $(12)$ for the representative item given by (13) \\
lp-I & $(14)-(15)$ \\
lp-MIID-EF & $(16)-(19)$ \\
lp-MIID-EF-R & $(16)-(19)$ for the representative item \\
lp-MIID-A-EF & $(20)-(23)$ \\
\hline
\end{tabular}

Table 5 displays the computational results obtained for lp, lp-A, lp-D, lp-R and lp-I. Each pair of columns (except the first one) give the value of the linear relaxation and running time. The best linear programming relaxation values were obtained for lp-D and lp-I relaxations. However lp-D has the disadvantage of using more execution time since it adds many more inequalities. 
Table 5. Computational results for the linear relaxation of the model (1)-(10) and the improvements from Section 3.1.

\begin{tabular}{ccccccccccc}
\hline & \multicolumn{2}{c}{ lp } & \multicolumn{2}{c}{ lp-A } & \multicolumn{2}{c}{ lp-D } & \multicolumn{2}{c}{ lp-R } & \multicolumn{2}{c}{ lp-I } \\
instance & value & time & value & time & value & time & value & time & value & time \\
\hline A11 & 1.4 & 0.9 & 20.0 & 1.4 & 20.9 & 91.1 & 20.0 & 0.9 & 20.5 & 0.9 \\
A21 & 1.4 & 0.8 & 20.0 & 1.3 & 21.0 & 97.7 & 20.0 & 0.9 & 20.5 & 1.0 \\
A31 & 1.4 & 0.9 & 20.0 & 1.4 & 20.9 & 109.3 & 20.0 & 0.9 & 20.5 & 0.9 \\
A12 & 1.4 & 0.7 & 20.3 & 3.7 & 22.7 & 79.8 & 20.1 & 0.8 & 25.7 & 0.7 \\
A22 & 1.4 & 0.7 & 20.2 & 3.4 & 22.5 & 101.1 & 20.0 & 0.6 & 25.8 & 0.7 \\
A32 & 1.4 & 0.7 & 20.2 & 3.7 & 22.7 & 101.2 & 20.0 & 0.6 & 23.9 & 0.7 \\
B11 & 1.4 & 0.9 & 20.0 & 1.4 & 20.9 & 93.6 & 20.0 & 0.9 & 20.5 & 0.9 \\
B21 & 1.4 & 0.8 & 20.0 & 1.3 & 21.0 & 106.7 & 20.0 & 0.8 & 20.5 & 1.0 \\
B31 & 1.4 & 0.9 & 20.0 & 1.4 & 20.9 & 120.1 & 20.0 & 0.9 & 20.5 & 0.9 \\
B12 & 1.4 & 0.7 & 20.3 & 3.7 & 22.7 & 79.1 & 20.1 & 0.8 & 25.7 & 0.7 \\
B22 & 1.4 & 0.7 & 20.2 & 3.3 & 22.5 & 91.6 & 20.0 & 0.6 & 25.9 & 0.7 \\
B32 & 1.4 & 0.7 & 20.3 & 3.3 & 22.7 & 105.3 & 20.0 & 0.6 & 29.6 & 0.7 \\
\hline
\end{tabular}

Table 6 displays the computational results for the linear relaxation of the extended formulations lp-MIID-EF, lp-MIID-EF-R, and lp-MIID-A-EF, see Table 4. As for Table 5, each pair of columns give the value of the linear relaxation of the improved model and the corresponding running time. The best value was obtained when considering model MIID-EF.

Table 6. Computational results for the linear relaxations of the extended formulations from Section 3.2.

\begin{tabular}{ccccccc}
\hline & \multicolumn{2}{c}{ lp-MIID-EF } & \multicolumn{2}{c}{ lp-MIID-EF-R } & \multicolumn{2}{c}{ lp-MIID-A-EF } \\
instance & value & time & value & time & value & time \\
\hline A11 & 23.3 & 3.9 & 20.0 & 0.9 & 20.0 & 0.9 \\
A21 & 23.7 & 3.8 & 20.0 & 0.9 & 20.0 & 0.9 \\
A31 & 23.4 & 5.6 & 20.0 & 0.9 & 20.0 & 0.9 \\
A12 & 25.7 & 8.3 & 20.3 & 1.0 & 20.3 & 1.0 \\
A22 & 26.2 & 7.6 & 20.4 & 1.1 & 20.4 & 1.1 \\
A32 & 26.4 & 7.4 & 20.4 & 1.0 & 20.4 & 1.0 \\
B11 & 23.3 & 3.9 & 20.0 & 0.8 & 20.0 & 0.8 \\
B21 & 23.7 & 3.8 & 20.0 & 0.8 & 20.0 & 0.9 \\
B31 & 23.4 & 5.5 & 20.0 & 0.8 & 20.0 & 0.9 \\
B12 & 25.6 & 8.1 & 20.4 & 1.0 & 20.4 & 1.0 \\
B22 & 26.2 & 6.7 & 20.4 & 1.2 & 20.4 & 1.2 \\
B32 & 25.7 & 9.5 & 20.4 & 1.1 & 20.4 & 1.0 \\
\hline
\end{tabular}

Overall, one can verify that the best approaches to improve the linear relaxation bound are the inclusion of the extended formulation, the disaggregated inequalities (12), lp-D relaxation, and the addition of inequalities (14) and (15), lp-I relaxation. While the two first cases imply a large increase on the size of the model, with inequalities (14) and (15) the size is kept under control.

Table 7 displays the computational results for Algorithm 1 and for the improved branchand-cut algorithm introduced in Section 5. The second and third columns give the objective 
value and running time of the first part of the heuristic scheme, that is, solving the relaxation model RMIID as described in Step 1 of Algorithm 1. The fourth column gives the value of the best solution found with the second part of the heuristic scheme as described in Steps 2 to 7 of Algorithm 1 and the corresponding running time is given in column five. The last three columns give the best solution value, the running time, and the final gap of the improved branch-and-cut procedure, Algorithm 2, solved with the Xpress solver and imposing a time limit of 1200 seconds.

Table 7. Computational results for the heuristic procedure, and for the improved branch-and-cut.

\begin{tabular}{|c|c|c|c|c|c|c|c|}
\hline \multirow[b]{2}{*}{ instance } & \multicolumn{4}{|c|}{ Heuristic } & \multicolumn{3}{|c|}{ Improved branch-and-cut } \\
\hline & $\begin{array}{l}\text { Relaxation } \\
\text { value }\end{array}$ & $\begin{array}{l}\text { RMIID } \\
\text { time }\end{array}$ & $\begin{array}{l}\text { Local } \\
\text { value }\end{array}$ & $\begin{array}{l}\text { ching } \\
\text { time }\end{array}$ & $\begin{array}{l}\operatorname{Imp} \\
\text { value }\end{array}$ & time & $\begin{array}{l}\text { cut } \\
\text { gap }\end{array}$ \\
\hline A11 & 34 & 100.4 & 34 & 57.7 & 34 & 5.9 & 0.0 \\
\hline A 21 & 37 & 99.6 & 37 & 100.0 & 37 & 6.5 & 0.0 \\
\hline A31 & 35 & 19.6 & 35 & 80.2 & 35 & 59.9 & 0.0 \\
\hline A12 & 38 & 99.9 & 38 & 100.2 & 38 & 218.9 & 0.0 \\
\hline A 22 & 39 & 71.9 & 39 & 100.5 & 39 & 1200 & 8.3 \\
\hline A32 & 37 & 38.0 & 37 & 103.2 & 37 & 204.8 & 0.0 \\
\hline B11 & 34 & 99.5 & 34 & 26.2 & 34 & 5.7 & 0.0 \\
\hline B21 & 37 & 100.3 & 37 & 101.4 & 37 & 6.1 & 0.0 \\
\hline B31 & 35 & 20.4 & 35 & 72.3 & 35 & 47.9 & 0.0 \\
\hline B12 & 39 & 99.4 & 38 & 100.0 & 38 & 366.7 & 0.0 \\
\hline B22 & 39 & 40.7 & 39 & 100.4 & 39 & 1200 & 8.5 \\
\hline B32 & 41 & 99.5 & 40 & 100.1 & 40 & 136.2 & 0.0 \\
\hline
\end{tabular}

With the improved branch-and-cut, the optimal value is obtained for 10 out of 12 instances. Only for the instances A22 and B22, the obtained solutions have a lower bound gap of 8.3 and 8.5, respectively. These results confirm both the quality of the heuristic and the importance of the improvements to derive optimal or near optimal solutions within reasonable running times.

\section{Conclusions}

The paper presents exact and heuristic approaches for a multi-item problem that combines lot-sizing and distribution decisions, occurring in the logistics of an hospital. All the approaches are based on a mathematical formulation which is improved with valid inequalities and an extended formulation. The heuristic scheme combines the resolution of a relaxation with a local search. The exact approach is a branch-and-cut algorithm based on the improved model and using as cut-off the upper bound provided with the heuristic scheme. Computational results show the heuristic and the exact approach are efficient in solving the tested instances.

For future research we intend to integrate the current weekly distribution planning models into a larger horizon planning problem where the orders from suppliers and the inventory management at the warehouse are considered.

\section{Acknowledgements}

The research of the first and third authors was partially supported through CIDMA and FCT, the Portuguese Foundation for Science and Technology, within project UID/MAT/ 
04106/2013. The research of the second author is financed by the ERDF - European Regional Development Fund through the Operational Programme for Competitiveness and Internationalisation - COMPETE 2020 Programme within project "POCI-01-0145-FEDER-006961", and by National Funds through the FCT as part of project UID/EEA/50014/2013.

\section{References}

1. Adulyasak, Y., Cordeau, J., Jans, R.: The production routing problem: A review of formulations and solution algorithms. Computers \& Operations Research 55, 141-152 (2015)

2. Agra, A., Andersson, H., Christiansen, M., Wolsey, L.: A maritime inventory routing problem: Discrete time formulations and valid inequalities. Networks 62, 297-314 (2013)

3. Agra, A., Christiansen, M., Delgado, A.: Mixed Integer Formulations for a Short Sea Fuel Oil Distribution Problem. Transportation Science 47, 108-124 (2013)

4. Agra, A., Christiansen, M., Delgado, A., Simonetti, L.: Hybrid heuristics for a short sea inventory routing problem. European Journal of Operational Research 236, 924-935 (2014)

5. Archetti, C., Bertazzi, L., Laporte, G., Speranza, M.G.: A branch-and-cut algorithm for a vendormanaged inventory-routing problem. Transportation Sci. 41(3), 382-391 (2007)

6. Atamtürk, A., Küçükyavuz, S.: Lot Sizing with Inventory Bounds and Fixed Costs: Polyhedral Study and Computation. Operations Research 53(4), 711-730 (2005)

7. Bijvank, M., Vis, IFA.: Inventory control for point-of-use locations in hospitals. Journal of the Operational Research Society 63, 497-510 (2012)

8. Brown, G., Keegan, J., Vigus, B., Wood, K.: The Kellogg company optimizes production, inventory, and distribution. Interfaces 31, 1-15 (2001).

9. Dacosta-Claro, I.: The performance of material management in health care organizations. The International Journal of Health Planning and Management, 17(1), 69-85 (2002)

10. de Vries, J.: The shaping of inventory systems in health services: A stakeholder analysis. International Journal of Production Economics, 133, 60-69 (2011)

11. Eppen, G.D., Martin, R.K.: Solving multi-item capacitated lot-sizing problems using variable redefinition. Operations Research 35, 832-848 (1997)

12. FICO Xpress Optimization Suite.

13. Fischetti, M., Lodi, A.: Local Branching. Mathematical Programming Ser. B 98, $23-47$ (2003)

14. Kelle, P., Woosley, J., Schneider, H.: Pharmaceutical supply chain specifics and inventory solutions for a hospital case. Operations Research for Health Care 1(2-3), 54-63 (2012)

15. Nemhauser, G., Wolsey, L.A.: Integer and Combinatorial Optimization. Wiley (1988)

16. Pan, ZX., Pokhareal, S.: Logistics in hospitals: a case study of some Singapore hospitals. Leadership in Health Services 20(3), 195-207 (2007)

17. Pochet, Y., Wolsey, L.A.: Production planning by mixed integer programming. Springer (2006)

18. Rosales, C.R., Magazine, M., Rao, U.: Point-of-Use Hybrid Inventory Policy for Hospitals. Decision Sciences 45(5), 913-937 (2014)

19. Rais, A., Viana, A.: Operations Research in Healthcare: a survey. International Transactions in Operational Research 18, 1-31 (2011)

20. Solyal, O., Sural, H.: A branch-and-cut algorithm using a strong formulation and an a priori tour-based heuristic for an inventory-routing problem. Transportation Sci. 45(3), 335-345. (2011)

21. Solyal, O., Sural, H.: The one-warehouse multi-retailer problem: Reformulation, classification and computational results. Annals of Operations Research 196(1), 517-541 (2012)

22. Uthayakumar, R., Priyan, S.: Pharmaceutical supply chain and inventory management strategies: Optimization for a pharmaceutical company and a hospital. Operations Research for Health Care 2, 52-64 (2013)

23. Volland, J., Fügener, A., Schoenfelder, J., Brunner, J.O.: Material Logistics in Hospitals: A Literature Review. (2015). 